\title{
Prognosis of Female Patients with Pulmonary Tuberculosis
}

\author{
Reiko SAKURAI, Ryuichiro SASAKI, Momoko YAMAGUCHI*, \\ Atsuko SHIBATA and Kunio AOKI
}

\begin{abstract}
To study the risk of death and causes of death among female patients with tuberculosis (TB), a total of 1,083 female patients who diagnosed as having active pulmonary TB and newly registered into the Nagoya TB registry between 1979-1981 were followed up till the end of 1983. During follow-up period 138 deaths $(\mathbf{1 2 . 7 \%})$ were observed, a significantly higher rate than expected from the general population. Observed deaths (O) from all types of cancer $(\mathrm{O} / \mathrm{E}$ ratio $=2.5)$, lung (6.4) and colorectal cancers (5.0), were significantly higher than that which was expected (E). The number of deaths from $\mathrm{TB}$ was naturally high $(\mathrm{O} / \mathrm{E}$ ratio $=40.0$, p <.001). Malignant lymphoma and multiple myeloma also showed high relative risk. The smoking rate among the patients was $14 \%$, about the same as with the general population. Excessive high rates of death from lung cancer were observed both among smoking and non-smoking cancer patients. Non-smokers showed a high proportion $(60 \%)$ of adenocarcinoma. The proportion of the use of antituberculous drugs during the past five years was not different between the lung cancer group and the non-lung cancer group. Patients who died from cirrhosis of the liver showed more frequent use of antituberculous drugs than the others. Factors causing excess incidence of colorectal cancer remain unknown. It was suggested that some immunologic disorders in TB patients may play some role in the development of cancer.
\end{abstract}

Key Words: Female tuberculosis, Cohort study, Lung cancer and other deaths due to cancer

Mortality from lung cancer in females has shown continual increase in Japan (1) despite a about $15 \%$ rate of smoking recorded over the last several decades (2). While adenocarcinoma has been identified as the main histologic type of female lung cancer noted in Japan (3), no satisfactory explanation, including passive smoking, outdoor air pollution or exposure to occupational hazards, has been obtained to explain this trend toward increasing female lung cancer rates. Recently, however, epidemiologic studies have indicated that preexistent non-cancerous pulmonary diseases may be associated with incidence of lung cancer $(4,5)$.

One of the authors, using retrospective cohort studies, observed in 1965 an excessively high incidence of lung cancer among male pulmonary tuberculosis (TB) patients registered in the city of
Philadelphia, $\mathrm{Pa}$. in the United States (4), but the risk of lung cancer among females was not examined in the study due to the small number of patients. Steinitz (5) has reported that there was a significantly higher risk of death due to lung cancer among pulmonary TB patients of both sexes in the TB registry in Israel, and that the risk of lung cancer among this population was as high as that of current smokers. The authors confirmed excessively high rates of death from lung cancer for both sexes among the pulmonary TB patients registered in Aichi Prefecture, Japan, during 1963-64 (6), the relative risks for males and females being 2.7-3.5 and $5.5-10.0$, respectively. No accurate examination of smoking history was made in that study, nor was any clinico-pathologic examination of cases of coexistent TB and lung cancer performed.

From Department of Preventive Medicine, Nagoya University School of Medicine, Nagoya and

* The National Institute of Nutrition, Tokyo

Received for publication January 24, 1989.

Reprint request to: Reiko Sakurai, MD, Department of Preventive Medicine,

Nagoya University School of Medicine, 65, Tsurumai-cho, Showa-ku, Nagoya 466, Japan 
The objective of this study is to examine excess risk of all types of cancer among female pulmonary TB patients listed in the Nagoya TB registry, as well as some of the causative factors affecting the incidence of cancer.

\section{SUBJECTS AND METHODS}

There were a total of 1,732 female patients newly registered into the Nagoya TB registry during the three year period from January 1, 1979 to December 31,1981 . Those patients with pleurisy (130 cases) and extrapulmonary TB without pulmonary lesions (152 cases) as well as those with lesions clinically healed with or without thoracic surgery ( 52 cases) were excluded from the study in order to allow the detection of new shadows indicating lung cancer using X-rays and the observation of the location of cancer lesions in relation to TB foci. No follow-up was performed regarding patients of 29 years of age or less (194 cases) or with primary TB infections (41 cases) as patients in these categories are generally too young to observe any occurrence of cancer. Cases which had been misdiagnosed as tuberculosis ( 80 cases) were also excluded. The remaining 1,083 cases, comprising those patients who had relapsed into pulmonary $\mathrm{TB}$, were followed up till the end of 1983.

These patients were given semiannual check-ups utilizing physician's reports made to public health centers, medical charts and home-visit records prepared by public health nurses. When a check-up schedule was interrupted either by the failure of the patient to show up the clinic or due to a change of address, inquiries were made through the public health nurse's office or via the local government to determine the vital status of the patient. When a patient had died, the cause of death was checked by obtaining a death certificate and/or medical records as well as pathological findings. When a case involved lung cancer, chest $\mathrm{X}$-rays as well as cytological and pathological findings were examined by the authors with the permission of attending physicians, in order to avoid misdiagnosis of other pulmonary diseases. Secondary cancer (metastasis) cases were carefully excluded from analyses of causes of death. Cases diagnosed as primary lung cancer within one year from entry into the TB registry were also omitted from the present analyses at the beginning of the study in order to exclude cases involving the simultaneous occurrence of a relapse into TB and lung cancer. Similar methods were also used to examine for the rates of incidence of other types of cancer.

To examine the effects of the severity or activity of TB lesions on the prognosis, patients with pulmonary TB were classified according to the presence of cavitary lesions and of Koch bacilli in sputum examination.

The use of antituberculous drugs by each patient was determined by referral to records maintained in the TB registries, each of which utilizes drugs such as Streptomycin (SM), INH, PAS and others. The smoking status of the patients and the use of antituberculous drugs were determined through referral to the examination records maintained by public health nurses, and the smoking habits of those patients with lung cancer were reexamined at the time of diagnosis and/or death, using clinical records. The rates of smoking among TB patients averaged $14.1 \%$, generally increasing with age (11.2\% for the 30-39 age group, $13.3 \%$ for $40-49$, $18.2 \%$ for $50-59,18.5 \%$ for $60-69$ and $16.4 \%$ for those over 70 ). A total of $10.8 \%$ of smokers exceeded a smoking index of 600 (number of cigarettes per day multiplied by years of smoking). There were a total of 911 non-smoking patients.

The expected number of deaths from each of the causes of death in this series was calculated using the person-year method based on age-sex specific death rates in the general population of Nagoya City in 1980 . The ratio of the observed number to the expected number of deaths (the $\mathrm{O} / \mathrm{E}$ ratio) was computed, and statistical significance was tested utilizing the Z-value obtained with the following formula:

$$
\mathrm{Z} \text {-value }=(\mathrm{O}-\mathrm{E}) / \sqrt{\mathrm{E}}
$$

When the expected number was less than ten, the $\mathrm{O} / \mathrm{E}$ ratio was tested by applying the Poisson distribution.

\section{RESULTS}

Table 1 shows the number of pulmonary TB patients and observation periods (person-years) by age at entry. About $70 \%$ of patients were 50 years old or older. The observation period totalled $3,845.75$ person-years, with 107 cases $(9.9 \%$ of the total) observable for less than two years. Observa- 
Table 1. The number of patients at risk and number of deaths among female patients with pulmonary tuberculosis registered in Nagoya in 1979-1981, by age at entry.

\begin{tabular}{|c|c|c|c|c|}
\hline Age & $\begin{array}{l}\text { Number of } \\
\text { patients }\end{array}$ & $\begin{array}{l}\text { Person-years } \\
\text { at risk }\end{array}$ & $\begin{array}{l}\text { Observed No. } \\
\text { of death }\end{array}$ & $\begin{array}{l}\text { Death rate } \\
\text { per } 100 \text { person-years }\end{array}$ \\
\hline $30-39$ & $153(14.1 \%)$ & 563.25 & 6 & 1.07 \\
\hline $40-49$ & $174(16.1 \%)$ & 627.75 & 6 & 0.96 \\
\hline $50-59$ & $214(19.8 \%)$ & 756. 50 & 18 & 2. 38 \\
\hline $60-69$ & $279(25.7 \%)$ & 969.25 & 29 & 3.00 \\
\hline $70-79$ & $192(17.7 \%)$ & 681.25 & 42 & 6.17 \\
\hline $80-$ & $71(6.6 \%)$ & 247.75 & 37 & 14.93 \\
\hline Total & $1.083(100 \%)$ & 3.845 .75 & 138 & 3. 59 \\
\hline
\end{tabular}

tion periods for the Koch bacilli positive and negative groups, and of those with and without cavitary lesions, totalled 1,097.00, 2,748.75, 1,078.25 and 2,767.50 person-years, respectively, and of nonsmoking patients, 3,238.00 person-years. The number of cases and amount of observed personyears for the over- 80 age group were smaller than those of the other groups.

The number of deaths and death rates per 100 person-years by age during the observation period are given in Table 1 . There were a total of 138 deaths during this period. The crude mortality rate totalling 3.59 per hundred person-years. The mortality rate increased with age, i.e., from 1.07 for the 30-39 age group to 14.93 for the group 80 years of age and over. These rates were all significantly higher than those of the general population. Mortality rates by type of disease were 3.7 per hundred person-years for the sputa positive group, 3.5 for the sputa negative group, and 4.1 and 3.4 , respectively for those with and without cavitary lesions. There was no significant difference noted between these groups.

The observed and expected number of deaths and $\mathrm{O} / \mathrm{E}$ ratios by cause of death are shown in Table 2. Significantly high rates of death from all causes were observed, with the $\mathrm{O} / \mathrm{E}$ ratio being calculated at 1.9. As was expected, patients with TB were at a very high risk of death from $\mathrm{TB}$ (with an $\mathrm{O} / \mathrm{E}$ ratio of 40.0). Furthermore, the $\mathrm{O} / \mathrm{E}$ ratios for cancer at all sites, heart disease, pneumonia and bronchitis and liver disease other than cancer were $2.5,2.4,3.2$ and 6.0 , respectively, all showing statistically
Table 2. Observed and expected numbers of deaths by causes of death among female patients with pulmonary tuberculosis registered in Nagoya in 1979-1981.

\begin{tabular}{|c|c|c|c|}
\hline Causes of death & $\begin{array}{l}\text { Observed } \\
\text { number }(0)\end{array}$ & $\begin{array}{l}\text { Expected } \\
\text { number (E) }\end{array}$ & $0 / E$ ratio \\
\hline A11 causes & $138(100.0 \%)$ & 73.0 & $1.9=\cdots$ \\
\hline TB, all forms & $12(8.7 \%)$ & 0.3 & $40.0^{* \cdots}$ \\
\hline Cancer, all sites & $31(22.5 \%)$ & 12.4 & $2.5^{\cdots}$ \\
\hline Heart diseases & $40(29.0 \%)$ & 16.7 & $2.4^{\cdots}=$ \\
\hline Cerebrovascular diseases & $14(10.1 \%)$ & 17.5 & 0.8 \\
\hline Pneumonia or bronchitis & $11(8.0 \%)$ & 3.4 & $3.2^{\cdots *}$ \\
\hline Liver diseases except cancer & $6(4.3 \%)$ & 1.0 & $6.0 \times *$ \\
\hline Other causes of death & $24(17.4 \%)$ & 16.9 & 1.4 \\
\hline
\end{tabular}

$* * * \mathrm{p}<0.001$

significance. A lower mortality ratio of 0.8 , however, was observed for cerebrovascular diseases, a figure which was not significantly low. The risk of death due to heart disease, pneumonia and bronchitis, and liver diseases other than cancer was slightly higher in cases with cavitary lesions than in those without. There was little difference in $\mathrm{O} / \mathrm{E}$ ratios between sputum positive and negative cases.

Table 3 shows the observed number of deaths and $\mathrm{O} / \mathrm{E}$ ratios from cancer by site. $\mathrm{O} / \mathrm{E}$ ratios for lung and colorectal cancers were 6.4 and 5.0, representing significantly higher figures, and groups with more active TB at entry showed relatively higher ratios than those with less active cases. It was noted that there was no death from cancer of the esophagus and stomach, and that there was a single death from liver cancer in this series. Higher risk of malignant lymphoma and multiple myeloma, 15.4 and 12.5 respectively was also noted, though only two deaths were noted for each cause.

The observed and expected numbers of deaths from lung cancer and the $\mathrm{O} / \mathrm{E}$ ratios in relation to smoking habits are shown in Table 4. The smoking rate among female TB patients in this series was $14.1 \%$, of which $86 \%$ quit smoking after the diagnosis of TB. The O/E ratio was very high 20.0 for smoking patients, a figure significantly higher than that of the general female population, corresponding ratio for non-smokers was 4.5.

There were nine cases who had both active TB 
Table 3. Observed number of death $(\mathrm{O})$ and $\mathrm{O} / \mathrm{E}$ ratios from cancer by site among female patients with pulmonary tuberculosis registered in Nagoya in 1979-1981, according to type of TB.

\begin{tabular}{|c|c|c|c|c|c|c|c|c|c|c|}
\hline \multirow[b]{3}{*}{ Causes of death } & \multicolumn{2}{|c|}{ Total } & \multicolumn{4}{|c|}{ Koch bacilli } & \multicolumn{4}{|c|}{ Cavity } \\
\hline & \multirow[b]{2}{*}{0} & \multirow[b]{2}{*}{ O/E } & \multicolumn{2}{|r|}{$(+)$} & \multicolumn{2}{|r|}{$(-)$} & \multicolumn{2}{|r|}{$(+)$} & \multicolumn{2}{|r|}{$(-)$} \\
\hline & & & 0 & $0 / E$ & 0 & $0 / E$ & 0 & O/E & 0 & $0 / E$ \\
\hline Cancer, all sites & 31 & $2.5^{* m *}$ & 10 & $2.5^{* *}$ & 21 & $2.2^{* * *}$ & 7 & 2.0 & 24 & 2. $5^{* * *}$ \\
\hline Lung & 9 & $6.4^{* * *}$ & 3 & $7.5^{* * *}$ & 6 & $6.3 \cdots$ & 3 & $8.2^{* * *}$ & 6 & $6.0 * *=$ \\
\hline Esophagus or stomach & 0 & - & 0 & - & 0 & - & 0 & - & 0 & - \\
\hline Colon or rectum & 6 & $5.0 *=x$ & 3 & $7.8^{* x x}$ & 3 & $3.2^{*}$ & 2 & $5.7^{* *}$ & 4 & $4.2^{=x}$ \\
\hline Liver & 1 & 1.6 & 0 & - & 1 & 2.2 & 0 & - & 1 & 2.2 \\
\hline Gallbladder or bile ducts & 2 & 2.1 & 1 & 3.6 & 1 & 1.5 & 0 & - & 2 & 2.9 \\
\hline Breast & 2 & 2.6 & 1 & 4.6 & 1 & 1.8 & 0 & - & 2 & $3.6^{*}$ \\
\hline Malignant 1ymphoma & & $15.4^{* * *}$ & 0 & - & & $22.2^{* * *}$ & & $33.3^{* * *}$ & & 10. $0^{* *}$ \\
\hline Multiple myeloma & & $12.5^{* * *}$ & 0 & - & & $18.2^{* * *}$ & 0 & - & & $16.7=* *$ \\
\hline Other forms of cancer & 7 & 1.6 & 2 & 1.5 & 5 & 1.6 & 1 & 0.9 & 6 & 1.9 \\
\hline
\end{tabular}

$* \mathrm{p}<0.05, * * \mathrm{p}<0.01, * * * \mathrm{p}<0.001$

Table 4. Observed and expected numbers of deaths from lung cancer and $\mathrm{O} / \mathrm{E}$ ratios among female patients with pulmonary tuberculosis registered in Nagoya in 1979-1981, with regard to the smoking status.

Status of smoking Observed number Expected number $0 / E$ ratio

\begin{tabular}{lllr}
\hline Smoker & 4 & 0.2 & $20.0^{* * *}$ \\
Non-smoker & 5 & 1.1 & $4.5^{* * *}$ \\
\hline Al1 & 9 & 1.4 & $6.4^{* * *}$ \\
\hline$* * * \mathrm{p}<0.001$ & & &
\end{tabular}

and cancer of the lungs, and one case of osteosarcoma of the thoracic wall was also discovered. Among the nine TB patients with lung cancer, four were smokers (two cases with squamous cell carcinoma, one of adenocarcinoma and one of small cell carcinoma), and five were non-smokers (two squamous cell carcinoma and three adenocarcinoma cases).

The frequency and duration of prescriptions for TB drugs, with the exception of SM, differed little between lung cancer patients and other non-cancer patients, for the series of TB patients who were prescribed such antituberculous drugs as INH, SM, Rifampicin (RFP) and Ethambutol (EB) for more than one year. SM was used in eight out of nine patients who died from lung cancer, while it was used in only $50 \%$ of non-cancer cases. Close scrutiny of these medical records revealed no difference in the histories of exposure to ionizing radiation indicated by medical diagnosis and treatment. The only differences noted being the different antituberculous drugs used to treat the nine lung cancer patients and the non-cancer patients.

\section{DISCUSSION}

In the present study, the patients with TB showed higher mortality rate than general population. Such differences may appear because of incomplete data collection in TB patients. Generally speaking, the reliability of these results depends on the accuracy of data, determined by the way of data collection and follow-up of these patients in the registry. Most of the newly diagnosed cases of TB in Japan are registered in the district where the patient resides, and diagnose of TB patients is carried out with the 
advice and direction of a local specialist committee established for that purpose, because the cost of treatment is borne in part by governmental subsidy and/or health insurance organizations. Each patient receives semiannual checkups within the registry by the specialist committee. Accordingly, the data collection and follow-up of TB patients seems to be satisfactory, as long as the treatment schedule is not interrupted. In this study newly registered patients were selected because previously existing cases were not so uniform in clinico-epidemiological characteristiçs in comparison with new cases. Because newly registered patients does not necessarily represent newly infected patients, there may be some possible biases.

The average duration of the follow-up conducted for this study was about four years, as that was the period of time over which an excessively high incidence of cancer was observed based on the study performed using the data from Philadelphia (4). Similar results were reported in a recent study conducted in Osaka, Japan (7).

In the present study, the unfavourable effects on mortality from some causes of death such as TB, all forms of cancer, heart diseases, pneumonia and bronchitis, and liver diseases except cancer were seen. The mortality from specific site of cancer such as lung, colorectal, malignant lymphoma, and multiple myeloma showed higher rate than unity.

The $\mathrm{O} / \mathrm{E}$ ratio of $\mathrm{TB}$ among TB patients was 40 times that of the general population. The analysis showed that those with more active $\mathrm{TB}$ lesions at entry into the registration showed a slightly higher mortality rate than the others, but no significant statistical differences. The patients with advanced TB lesions in the lung showed higher mortality rate from heart diseases and pneumonia and bronchitis. These results reflects the extent of TB lesion.

No excessively high rate of death due to liver cancer was noted, though the death rate from cirrhosis of the liver was significantly high. The record linkage study of registered TB patients in the Kyushu area in Japan showed an excessive death rate from cirrhosis of the liver as well (8). Recent reports show that the use of $\mathrm{INH}$ in chemoprevention results in the reduced liver function (9-13). Prolonged use of antituberculous drugs may bear a relation to the excessive death rate in cases of cirrhosis of the liver.
In the Philadelphia study (4), however, no excessively high death rate from liver diseases or cancer of the colon among Caucasians was observed. To study the relation between TB and liver and/or colon cancer, further clinico-epidemiological study will be need.

The number of newly registered TB patients in Japan (14) has gradually decreased to about 17 thousand by 1970 and 7.1 thousand by 1980 . The reduction for the age groups over 60 years has been less, however, due to the increasing numbers of aged people in this country, and was only $10 \%$ for the last decade. The authors have attempted to examine the occurrence of cancer among female pulmonary TB patients newly registered into the Nagoya TB registry and to discuss possible etiological factors related to cancer other than smoking.

In this study, thirteen cases of lung cancer diagnosed within one year after TB registration were excluded, as it is generally difficult to judge which lesion occurred first. An additional case of lung cancer was excluded from the data examined for this study as the patient was still alive at the close of the observation period. Even after this exclusion, the relative risk from cancer of lung among TB patients showed significantly high risk.

While lung cancer was closely related to smoking, non-smoking patients in this series also showed a risk of 4.5 , significantly higher than that of the general population. Passive smoking may be another factor affecting the incidence of lung cancer: the reported risk among "passive smoking" wives was about 2.0 (15), about half as high as the above-mentioned TB group's risk. A total of 3,579 female pulmonary TB patients in the Aichi Prefecture TB registry during 1963-64 (6) were followed up in the past for a five year period, during which time the relative risk was determined to be 5.5 overall, but in this study the risk among non-smokers could not be analyzed. Even considering indoor air pollution and exposure to other hazardous agents, this higher risk in females defies satisfactory explanation, leading to a hypothesis that there may be some other as yet undiscovered factors. Similar studies conducted on TB patients registered in other areas of Japan $(7,8,16$, 17) showed a higher mortality rate of lung cancer, although there were no clinico-pathological examinations at the time of cancer diagnosis or at death. 
In the autopsy series, case of lung cancer death in Japan during the last two decades showed a significantly higher rate of advanced TB than did the cases who died from cerebrovascular diseases or gastric cancer (18).

Lung cancer foci tended to appear in the lung field without TB lesions even when in the same lobe where TB lesions had previously existed. The frequency of cancer lesions associated with TB scars seemed to be low in the Philadelphia study (4). INH is also reported to act as a carcinogenic agent in experimental animals. Still, antituberculous drug regimens during the period of five year's follow-up, including INH, were similar, though past history of INH use prior to registration could not be examined. Three studies on the effects of INH on lung cancer among TB patients in Japan $(17,19,20)$ showed no causal relationship between INH and lung cancer. Cohort studies of industrial workers employed by the Japan National Railroad Enterprise revealed no association between INH and lung cancer among pulmonary TB patients (17), although they did indicate the existence of a significantly high incidence of lung cancer within the entire group. The high proportion of SM use among the cases who died from lung cancer, when compared with the controls, is currently difficult to explain because of the lack of information regarding the carcinogenicity of SM.

A history of exposure to ionizing radiation for medical diagnosis and treatment revealed no excessive risk among cancer cases and non-cancer cases, and a history of radiological treatment to the pneumothorax revealed no excessive risk of lung cancer (21). A higher incidence of lung cancer was observed among patients with sarcoidosis (22) and pulmonary fibrosis (23), and among patients with immunodeficiencies such as dermatomyositis and progressive systemic sclerosis (24) with pulmonary involvement. Considering the extended latency period of cancer and other clinico-pathological features of lung cancer, TB foci themselves do not seem to be directly associated with the occurrence of lung cancer. Some specific host conditions such as immunological disorders already in existence at the time of exacerbation or relapse of TB lesions or in the manifestation of diseases may relate to the promotion of the development of cancer, and the risk was high during the first four years after the observation period started. As stated previously, 13 cases were diagnosed as lung cancer within one year after TB registration. It should also be noted that the clinical onset of lung cancer may relate to immunological status of the host because of higher mortality from malignant lymphoma and multiple myeloma which are closely associated with immunological disorders. Considering the fact that an excessively high incidence of cancer among relapsed, active TB patients was observed during first four years of follow-up in this study, it may be possible to say that some immunological disorders of the host may be related to the development of cancer.

It was not expected that this study would indicate an excessively high risk of colorectal cancer. There were no differences in the uses of antituberculous drugs, radiation or other medical procedures between cases of colorectal cancer and non-cancer cases. On the other hand, there were no cases of esophageal or stomach cancer. Japanese-Americans in Hawaii experienced higher incidence of colorectal cancer, the level of which was about the same as that of Caucasians, while the incidence of stomach and esophageal cancers was lower (25). This result indicates that diet may play some role in the incidence of colorectal cancer, as this group tends to consume more Western-style cuisine than do ethnic Japanese living in Japan.

ACKNOWLEDGEMENTS: Authors gratefully acknowledge the assistance of Mr. S. Mizuno for his help in the statistical analysis of the data for this reports, and of also thank gratefully Drs. K. Yamanaka, M. Fujimoto and T. Nakata for their most helpful and appropriate assistance and advice in the preparation of the report.

\section{REFERENCES}

1) Statistics and Information Department, Ministry's Secretariat, Ministry of Health and Welfare (ed): Vital Statistics in Japan, 1947-1984, Kosei-Tokei Kyokai, Tokyo, 1949-1985 (in Japanese).

2) Tominaga S: Smoking in Japan. in: The UICC Smoking Control Workshop, Tominaga S, Aoki K, Eds. Univ Nagoya Press, Nagoya, 1982, p.27.

3) Shimizu H: Comparisons of lung cancer incidence rates between Japan and U.S.A. by sex and histologic type. Lung Cancer (Haigan) 21: 519, 1981 (in Japanese).

4) Aoki K, Ipsen J Jr, Stern SC: Studies on the epidemiology of lung cancer in relation to pulmonary tuberculosis. in: Epidemiological Aspects of Tuber- 
culosis in Israel and in Japan, Klingberg MA, Turner I, Eds. Israel Inst Biological Res Ness-Ziona, 1969, p.30.

5) Steinitz R: Pulmonary tuberculosis and carcinoma of the lung. A survey from two population-based disease registers. Am Rev Respir Dis 92: 758, 1965.

6) Aoki K, Sasaki R, Hamajima N, et al: Epidemiology of lung cancer and tuberculosis. in: Cancer Prevention in Developing Countries, Kyogali M, Omar YT, Eds. Pergamon Press, Oxford, 1986, p.171.

7) Takatorige $T$, Nakamura $T$, Ueda $H$, et al: Follow-up study on the registered tuberculosis patients-analysis of causes of death, J Public Health $\mathbf{3 0}$ (Specific issue for the Annual Meeting): 218, 1983 (in Japanese).

8) Hongo N, Kido $\mathrm{H}$, Aoki $\mathrm{M}$, et al: Causes of death among the tuberculosis patients registered - An investigation in the Kyushu district. Kekkaku 56: 540, 1981 (in Japanese).

9) Smith JP, Scharer L: Adverse effects of isoniazed and their significance for chemoprophylaxis. Am Rev Resp Dis 102: 821, 1970.

10) Garibari RA, Drusin RE, Ferebee SH, et al: Isoniazidassociated hepatitis. Reports of outbreak. Am Rev Respir Dis 106: 357, 1972.

11) Kopanoff $\mathrm{DE}$, Snider DE, Caras GJ: Isoniazid related hepatitis. A U.S. public health service cooperative surveillance study. Am Rev Respir Dis 117: 991, 1978.

12) Blach M, Mitchell JR, Zimmerman MJ, et al: Isoniazid associated hepatitis in 114 patients. Gastroenterology 69: $289,1975$.

13) Comstock GW, Edwards PQ: The competing risks of tuberculosis and hepatitis for adult tuberculin reactors. Am Rev Respir Dis 111: 573, 1975.

14) Ministry of Health and Welfare, Health Service Bureau, Tuberculosis Intractable Diseases and Infectious Diseases Division (ed: Statistic of Tuberculosis 1965-1985, Kekkaku Yobokai, Tokyo, 1965-85.

15) Hirayama T: Non-smoking wives of heavy smokers have a higher risk of lung cancer: A study from Japan. Br Med J 282: 183, 1981.

16) Watanabe $\mathrm{H}$ : Current status of cancer deaths in a small area. in: Report of the Research Committee on Epidemiology of Cancer Supported by Ministry of
Health and Welfare (Chairman Hirayama T), 1977 (in Japanese).

17) Fukuta A: Report of the Research Committee on Early Detection of Lung Cancer in Specific Environments Supported by Ministry of Health and Welfare (Chairman Iwasaki T), 1971 (in Japanese).

18) Aoki K, Ohtani M: Frequency of Pulmonary Tuberculosis and Silicosis in Lung Cancer Cases Autopsied in Japan Based on the Annuals of Pathological Autopsy. Division of Epidemiology, Aichi Cancer Center Research Institute, Nagoya, 1971 (in Japanese).

19) Shimamura K: Study on correlation between INH and lung cancer. in: Report of the Research Committee on Early Detection of Lung Cancer in Specific Environments Supported by Ministry of Health and Welfare (Chairman Iwasaki T), 1973.

20) Aoki K: Epidemiologic study on lung cancer among the tuberculosis patients. in: ibid, 1971-1973.

21) Iwasaki T, Aoki M: Study on incidence of cancer among the highly exposed to ionizing radiation by medical diagnosis and treatments. in: ibid, 1973.

22) Brincker $\mathrm{H}$, Wilbek $\mathrm{E}$ : The incidence of malignant tumors in patients with respiratory sarcoidosis. $\mathrm{Br} \mathrm{J}$ Cancer 29: 247, 1974.

23) Yamaoka M, Fukuchi Y, Ishida Y, et al: Study on clinico-pathological features and prognosis of idiopathic interstitial pulmonary fibrosis - Analysis of 500 cases from nation-wide survey. in: Reports of Research Committee on Interstitial Pulmonary Fibrosis Supported by Ministry of Health and Welfare of Japan (Chairman Harasawa M), Department of Geriatrics, Univ of Tokyo, 1984, p.5,

24) Shimamine $T$ : Statistical arrangement of epidemiological uses of autopsy cases from the Annuals of Pathological Autopsy-Collagen diseases and cancer. in: Reports at the Second Annual Meeting of the Research Committee on Epidemiology of Intractable Diseases Supported by Ministry of Health and Welfare of Japan (Chairman Uematsu M), 1981.

25) Doll R, Peto R: The causes of cancer: Quantitative estimates of avoidable risks of cancer in the United States today. J Natl Cancer Inst 66: 1191, 1981. 